\title{
A semantic constraint on binary determiners*
}

\author{
R. Zuber, CNRS, Paris
}

\begin{abstract}
A type $\left\langle 1^{2}, 1\right\rangle$ quantifier $F$ is symmetric iff $F(X, X)(Y)=F(Y, Y)(X)$. It is shown that quantifiers denoted by irreducible binary determiners in natural languages are both conservative and symmetric and not only conservative.
\end{abstract}

\section{Introduction}

It is generally admitted that in natural languages nominal unary determiners, that is functional expressions which form noun phrases when applied to a common noun, denote not arbitrarily type $\langle 1,1\rangle$ quantifiers (binary relations between sets) but only those which satisfy the constraint of conservativity. This constraint, stated somewhat imprecisely, indicates that to determine the truth conditions of sentences with such determiners it is not necessary to take into account all sets determined by the arguments of the function; in particular the complement of the first set-theoretic argument does not matter. It follows from this that some type $\langle 1,1\rangle$ quantifiers are not "naturally" denotable even by complex unary determiners.

The conservativity of natural language determiners is sometimes considered as a language universal: all types of determiners in all natural languages are conservative in the sense that they denote only conservative functions. Even though some non-conservative determiners are known, it appears that they are rare and not arbitrary since they are systematically related to conservative determiners (cf. Zuber 2004a).

It has also been established that NLs display binary or even n-ary determiners, that is functional expressions which form noun phrases with more than one common noun (cf. Keenan and Moss 1985). A simple example of such a binary determiner is given by the comparative determiner more... than as it occurs in the noun phrase more students than teachers. Such n-ary determiners denote higher type quantifiers which are $n+1$-ary relations between sets.

* The basic content of this paper was presented at The Semantics Research Group meeting in Tokyo, in April 2008. Thanks to Makoto Kanazawa, Ed Keenan, Ross Charnock, Maryvonne Daguenet-Teissier Bernard Teissier and the referee of the journal for some corrections and suggestions. Makoto in particular suggested a simplification of the definition of symmetric quantifiers and Maryvonne and Bernard helped me with some combinatoric problems. Ross, as usually, corrected my English 
The notion of conservativity has been extensively discussed mainly in the context of unary determiners and type $\langle 1,1\rangle$ quantifiers (Keenan and Stavi 1986, Keenan and Westerståhl 1997, Peters and Westerståhl 2006). However, it easily generalises to higher type quantifiers denoted by n-ary determiners. Consequently the universalistic claim concerning conservativity of $n$-ary determiners extends implicitly as well. Thus one considers as a language universal the claim that n-ary, or more specifically binary, determiners in all NLs are conservative.

Since higher type quantifiers take more arguments than "simple" type $\langle 1,1\rangle$ quantifiers, there are obviously "many more" higher type quantifiers. For instance in the finite universe with $n$ elements there are $2^{4^{n}}$ of all type $\langle 1,1\rangle$ quantifiers and $2^{8^{n}}$ of all type $\langle 1,1,1\rangle$ quantifiers (that is functions from 3 set arguments to truth-values). A striking empirical observation is, however, that the set, or at least the number of patterns, of expressions denoting type $\langle 1,1,1\rangle$ quantifiers seems to be very limited, as we will see. This means very likely that there are additional constraints governing the semantics of such "higher type" determiners.

The purpose of this paper is to show that binary determiners in addition to conservativity satisfy the natural constraint of symmetry. In the case of unary determiners symmetry just means that in simple sentences with such determiners one can permute verbal and nominal arguments without changing the truth-value of the whole. The notion of symmetry can be generalised to quantifiers denoted by binary (or even n-ary) determiners (Zuber 2007). It appears than that a huge majority of binary determiners, if not all of them, denote symmetric quantifiers. For instance the comparative determiner more...than denotes a symmetric quantifier because in particular sentences in (1) are equivalent:

(1a) More students than teachers are Buddhists.

(1b) More Buddhists are students than teachers.

So in this paper we are interested in a sub-set of monadic quantifiers, that is, specific relations between sets. A unary monadic quantifier, or type $\langle 1\rangle$ quantifier, is a function from sets to truth-values. It is a denotation of a noun phrase (used in subject position in a sentence). The type of $n+1$-ary monadic quantifiers is often noted as $\langle 1,1, \ldots, 1\rangle$ ("n+1-times" "1") or $\left\langle 1^{n+1}\right\rangle$. We will not quite follow this notation. In order to distinguish between "nominal" (or "restrictive") and "verbal" (or "predicative") arguments of a quantifier we will make a difference between type $\left\langle 1^{n}, 1\right\rangle$ and type $\left\langle 1,1^{n}\right\rangle n+1$-ary monadic quantifiers. The former class corresponds to monadic quantifiers which have $n$ nominal arguments and one verbal argument whereas the latter class corresponds to monadic quantifiers which have one nominal argument and $n$ verbal arguments. This notation partially indicates different structure ("syntax") of expressions denoting quantifiers. More importantly, it accounts for the fact that some properties of quantifiers may depend on the type - nominal or verbal- of 
the argument. We will see, for instance, that the cardinality of conservative type $\left\langle 1^{2}, 1\right\rangle$ quantifiers is not the same as the cardinality of conservative type $\left\langle 1,1^{2}\right\rangle$ quantifiers.

Binary monadic quantifiers, that is type $\langle 1,1\rangle$ quantifiers, will be called simple quantifiers. Quantifiers of type $\left\langle 1^{n}, 1\right\rangle$ or of type $\left\langle 1,1^{n}\right\rangle$, for $n>1$, will be called higher type quantifiers.

Final introductory remark. As already stated, we will distinguish quantifiers from determiners. Determiners, as understood here, are linguistic expressions which denote quantifiers or give rise to other expressions denoting quantifiers. They are functional expressions, often discontinuous, which apply to one or many arguments of the same category. Their arguments all denote either nominal or verbal arguments of a quantifier. This means that the arity of a determiner is one less than the arity of the quantifier they denote. Thus a unary determiner is a functional expression taking one argument, a common noun. It denotes a binary monadic quantifier since it induces a binary relation between the denotation of its argument and the denotation of the verb phrase of the sentence in which it occurs. For instance the determiner all denotes a simple quantifier which corresponds to a binary relation $A L L$ between sets such that $A L L(X)(Y)$ holds iff $X \subseteq Y$. Similarly the binary determiner more... than..., when occurring in a NP on subject position, denotes a ternary monadic type $\left\langle 1^{2}, 1\right\rangle$ quantifier since its two "nominal" arguments denote sets and the verb phrase of the sentence in which the determiner occurs (in the NP on the subject position) also denotes a set (as assumed here).

As we will see, the syntactic status of determiners is not always so clear, in particular with respect to binary determiners taking verbal arguments (the so-called identity comparatives discussed in section 4 below). This fact does not prevent us, however, from making some semantic generalisations based on clearer syntactic cases.

The paper is organised as follows. First, I will recall some basic properties of simple, type $\langle 1,1\rangle$, quantifiers, focusing on symmetric ones, and their duals, contrapositional quantifiers. It will be generally assumed that the universe of discourse is finite. Then in the next section I will show how various properties, in particular conservativity and symmetry, extend to higher type quantifiers. Finally various known types of binary determiners will be examined in order to show that all of them are symmetric (denote symmetric quantifiers). All this will be done using the framework of Boolean semantics (Keenan and Faltz 1986) since, as will be shown, various involved classes of quantifiers have the Boolean structure. This fact will be used to make more precise various formal claims about denotations of binary determiners. They will be stated precisely though (usually simple) proofs will be omitted

\section{Varieties of simple quantifiers}

In this section we are interested in the denotations of (unary) nominal determiners. These are expressions (like every, no, some...including Lea, most) 
which combine with common nouns to form noun phrases. Thus, semantically, they are functions from $P(E)$ onto type $\langle 1\rangle$ quantifiers, where $E$ is the universe of discourse and a type $\langle 1\rangle$ quantifier is a set of sub-sets of $E$. They are type $\langle 1,1\rangle$ quantifiers and will be called here simple quantifiers. These quantifiers can be viewed as binary relations on sets. Indeed a type $\langle 1,1\rangle$ quantifier $F$, which is a function in $[P(E) \rightarrow[P(E) \rightarrow\{0,1\}]]$, corresponds to the binary relation $Q$ between sets defined by $Q X Y \Leftrightarrow F(X)(Y)=1$. The set of all type $\langle 1,1\rangle$ quantifiers, or the set of unrestricted functions belonging to $[P(E) \rightarrow[P(E) \rightarrow\{0,1\}]]$ will be denoted by $P D E T$. This set forms an atomic Boolean algebra. Any member $F$ of $P D E T$ has a Boolean complement $\neg F$ and a post-complement $F \neg$ defined in the usual way.

It has been noticed that the class PDET is too "large" to be the set of possible denotations for unary determiners since all functions denoted by such determiners satisfy various constraints. One of the best known such constraints on possible denotations of determiners is conservativity. By definition:

D1: $F$ is conservative or $F \in C O N S$ iff for any property $X, Y$ and $Z$ if $X \cap Y=X \cap Z$ then $F(X)(Y)=F(X)(Z)$

Conservativity of type $\langle 1,1\rangle$ quantifiers can additionally be formulated in two different ways:

Fact 1 (cf. Keenan and Faltz 1986) $F \in C O N S$ iff for any property $X, Y$ one has $F(X)(Y)=F(X)(X \cap Y)$

Fact 2 (Zuber 2005): $F \in C O N S$ iff for any property $X, Y$ one has $F(X)(Y)=$ $F(X)\left(X^{\prime} \cup Y\right)$

The constraint of conservativity considerable reduces the number of conservative functions in comparison with the number of unrestricted functions. Thus we have (Beghelli 1992):

Proposition 3: If $|E|=n$ then $|P D E T|=2^{4^{n}}$ and $|C O N S|=2^{3^{n}}$

It follows from Proposition 3 that in the universe with just two elements we have 65,536 of unrestricted type $\langle 1,1\rangle$ quantifiers among which there are only 512 conservative ones.

There are various empirically and theoretically important sub-classes of the $C O N S$ algebra. Thus CONS has two sub-algebras, the algebra INT of intersective functions, and the algebra $C O-I N T$ of co-intersective functions (Keenan 1993). By definition:

D2: $F \in I N T$, iff for all properties $X, Y, Z$ and $W$, if $X \cap Y=Z \cap W$ then $F(X)(Y)=F(Z)(W)$.

D3: $F \in C O-I N T$ iff for all properties $X, Y, Z$ and $W$, if $X-Y=Z-W$ then $F(X)(Y)=F(Z)(W)$. 
Intersective and co-intersective type $\langle 1,1\rangle$ quantifiers can be defined in four equivalent ways as indicated by the following facts (Zuber 2007):

Fact 4: The following four conditions are equivalent:

(i) $F \in I N T$

(ii) $F(X)(Y)=F(X \cap Y)(X \cap Y)$

(iii) $F(X)(Y)=F(E)(X \cap Y)$

(iv) $F(X)(Y)=F(X \cap Y)(E)$

Fact 5: The following four conditions are equivalent:

(i) $F \in C O-I N T$

(ii) $F(X)(Y)=F(X-Y)\left(X^{\prime} \cup Y\right)$

(iii) $F(X)(Y)=F(X-Y)(\emptyset)$

(iv) $F(X)(Y)=F(E)\left(X^{\prime} \cup Y\right)$

Both sets, INT and CO-INT, form atomic (and complete) Boolean algebras (sub-algebras of CONS). Atoms of INT and of CO-INT are determined by a property. Exclusion determiners denote atoms of these algebras: no...except Leo and Lea denotes an atom of the algebra of intersective function determined by the set composed of two elements, Leo and Lea (Zuber 1998).

The algebra $I N T$ contains a sub-algebra $C A R D$ of cardinal determiners: they are denotations of, roughly speaking, various numerals. By definition:

D4: $F \in C A R D$ iff for all properties $X, Y, W$ and $Z$, if $|X \cap Y|=|W \cap Z|$ then $F(X)(Y)=F(W)(Z)$.

Any cardinal number determines an atom of $C A R D$. The determiner exactly $n$ denotes an atomic cardinal function.

As might be expected the algebra $C O-I N T$ has an analogous sub-algebra. This is the algebra CO-CARD of co-cardinal functions (Keenan 1993):

D5: $F \in C O-C A R D$ iff for all properties $X, Y, W$ and $Z$, if $|X-Y|=|W-Z|$ then $F(X)(Y)=F(W)(Z)$

Determiners like every...except five denote co-cardinal functions. Moreover, every post-complement of a cardinal quantifier is a co-cardinal quantifier.

Unary determiners whose semantics also involves cardinality of sets denoted by their arguments are those denoting proportional quantifiers PROPORT. They are defined as follows (Keenan 2002):

D6: $F \in P R O P O R T$ iff for all sets $X, Y, W, Z \subseteq E$ if $|W| \times|X \cap Y|=$ $|X| \times|W \cap Z|$ then $F(X)(Y)=F(W)(Z)$

Proportional quantifiers form an atomic Boolean algebra (Zuber 2005).

A classical example of a proportional quantifier is the quantifier denoted by 
the determiner most (in the sense of more than half).

Let me mention in addition the algebra of so-called generalised cardinals or GCARD introduced in Keenan and Faltz 1975 (under the name of cardinality dependent) and, independently in Zuber 2004b and studied in more detail in Zuber 2005. By definition:

D7 $F \in G C A R D$ iff for all properties $X, Y, Z$ if $|X \cap Y|=|X \cap Z|$ then $F(X)(Y)=F(X)(Z)$.

What definition D7 says intuitively is that a generalised cardinal is a function which cannot distinguish among properties $Y_{1}$ and $Y_{2}$ at the argument $X$ if $X \cap Y_{1}$ and $X \cap Y_{2}$ have the same cardinality.

Obviously the algebra GCARD is a proper sub-algebra of $C O N S$ and contains as proper sub-algebras $C A R D$ and, only in finite universes, $C O-C A R D$. Moreover the following fact is also true (Zuber 2005):

Fact 6: PROPORT is a sub-algebra of GCARD.

Proof: It is enough to show that any proportional quantifier is a generalised cardinal. Suppose that $D$ is proportional and that for arbitrary $X, Y$ and $Z$ one has $|X \cap Y|=|X \cap Z|$. Then it is also true that $|X| \times|X \cap Y|=|X| \times|X \cap Z|$. Since $D$ is proportional this means that $F(X)(Y)=F(X)(Z)$ and thus $D$ is generalised cardinal.

I mentioned the algebra $G C A R D$ because, as indicated by above properties, many classes of conservative quantifiers are generalised cardinals. Let us see some examples. Quantifiers $N O$ and FIVE are generalised cardinals because they are cardinals. Similarly EVERY and EVERY...EXCEPT 10 are generalised cardinals because they are co-cardinals. Notice that this last claim is true only for finite universes since only in this case is it true that $\left|X \cap Y_{1}\right|=\left|X \cap Y_{2}\right|$ iff $\left|X \cap Y_{1}^{\prime}\right|=\left|X \cap Y_{2}^{\prime}\right|$ (where $Y_{1}^{\prime}$ and $Y_{2}^{\prime}$ are Boolean complements of $Y_{1}$ and $Y_{2}$ respectively).

Consider now the determiner the $n$. It denotes the quantifier $T H E n$ defined as follows: $T H E n(X)(Y)=1$ iff $|X|=n$ and $X \subseteq Y$. Observe that THE $n$ is neither cardinal nor co-cardinal. One can also check that it is not proportional: to see this (for $n=1$ ) take $X, Y, W, Z$ such that $|X|=1,|W|=2, X \subseteq Y$ and $W \subseteq Z$. It is easy to see, however, that the $n$ denotes a generalised cardinal quantifier.

Similarly one can show that the determiners like most but less than 10 or most or at least 10 denote (proper, that is neither cardinal, nor co-cardinal, nor proportional) generalised cardinals. We show now that the quantifier MOST $O R A T-L E A S T(10)$ is neither cardinal nor proportional. We show first that it is not cardinal. Suppose for this that $|X \cap Y|=|W \cap Z|<10,|X \cap Y| \leq\left|X \cap Y^{\prime}\right|$ and $|W \cap Z|>\left|W \cap Z^{\prime}\right|$. In this case $M O S T(X) O R A T-L E A S T(10)(X)(Y)=$ 0 and $\operatorname{MOST}(W)$ OR AT-LEAST $(10)(W)(Z)=1$ which means that MOST 
OR AT-LEAST(10) is not cardinal.

Suppose now that $|X \cap Y|=\left|X \cap Y^{\prime}\right|=10,|W \cap Z|=8,|X|=20$ and $|W|=16$. In this case $|W \times| X \cap Y|=| X|\times| W \cap Z \mid$. However in this case $\operatorname{MOST}(X)$ OR AT-LEAST $(10)(X)(Y)=1$ and $M O S T(W) O R A T$ $\operatorname{LEAST}(10)(W)(Z)=0$ which means that the considered quantifier is not proportional. It is, however, a generalised cardinal because it is a join of two generalised cardinals.

The important point in the context of unary determiners in the above examples is that not all of them denote generalised cardinals. To see this it is enough to take a properly intersective (non-cardinal) quantifier or a properly co-intersective (non-co-cardinal) quantifier. For instance No...except Leo, most/some ...including Leo and every... except Leo are not generalised cardinals. We will see, however, that many binary determiners denote (appropriately generalised to the higher type case) generalised cardinals.

We can now introduce two other classes of type $\langle 1,1\rangle$ quantifiers: symmetric and contrapositional ones. When extended to higher type quantifiers they will play an essential role in our analysis of binary determiners. In addition they allow us to better understand the relationship between conservative quantifiers in general and their various sub-classes.

Symmetric and contrapositional type $\langle 1,1\rangle$ quantifiers are defined as follows (Zuber 2007):

D8: $F \in S Y M$ iff for all properties $X, Y$ one has $F(X)(Y)=F(Y)(X)$

D9: $F \in C O N T R$ iff for all sets $X, Y$ one has $F(X)(Y)=F\left(Y^{\prime}\right)\left(X^{\prime}\right)$.

Both sets, $S Y M$ and CONTR form atomic Boolean algebras. Their elements need not to be conservative. In addition $S Y M$ and $C O N T R$ are not disjoint. For instance for any set $A$, the set of pairs $\left\{\langle A, A\rangle,\left\langle A^{\prime}, A^{\prime}\right\rangle\right\}$ corresponds to a non-conservative simple quantifier which is symmetric and contrapositional at the same time.

The following propositions show when symmetric and contrapositional quantifiers are conservative (Zuber 2007):

Proposition 7: $C O N S \cap S Y M=I N T$

Proposition 8: $C O N S \cap C O N T R=C O-I N T$

It follows from Propositions 7 and 8 that under conservativity type $\langle 1,1\rangle$ symmetric quantifiers are the same as the intersective ones and contrapositional quantifiers are the same as co-intersective ones. As we will see this is not the case for higher order quantifiers.

Interestingly, we have similar relations between algebras $G C A R D, C A R D$, $C O-C A R D$ and $S Y M$ and CONTR. More precisely, the following propositions hold (Zuber 2007):

Proposition 9: $G C A R D \cap S Y M=C A R D$ 
Thus cardinal and intersective quantifiers are symmetric. For example FIVE, SOME, SOME..., INCLUDING LEA and NO, ,., EXCEPT LEO are symmetric quantifiers.

It is interesting that one can define symmetric quantifiers in the format we use in other definitions and which can be easily generalised to quantifiers of higher types. The following trivial proposition which indicates such an equivalent definition will be used as a convenient handle for generalising symmetry to higher types (Zuber 2007) :

Proposition 11: $F \in S Y M$ iff there exists a commutative binary function " $\otimes$ " taking sets as arguments such that for all $X, Y, W, Z$ if $X \otimes Y=W \otimes Z$ then $F(X)(Y)=F(W)(Z)$.

A similar property holds for contrapositional quantifiers:

Proposition 12: $F \in C O N T R$ iff there exists a commutative binary function $" \otimes "$ taking sets as arguments such that for all $X, Y, W, Z$ if $X \otimes Y^{\prime}=W \otimes Z^{\prime}$ then $F(X)(Y)=F(W)(Z)$.

Propositions 11 and 12 will allow us to generalise the notion of symmetry and contraposition to quantifiers of higher types. We will see, however, that in this case the distinction between symmetry and contraposition disappears.

\section{$3 \quad$ Quantifiers of higher types}

In the previous section we presented various properties of type $\langle 1,1\rangle$ quantifiers. They are denotations of unary determiners. Since we are going to make some claims about constraints on denotations of binary determiners we need to consider how to extend various properties discussed in the previous section to a more general case of higher type quantifiers. Though we are basically interested in denotations of binary determiners, in most definitions we propose we will not limit the number of arguments corresponding to the arguments of the determiner. Thus we define various properties of higher type quantifiers so that they are applicable to denotations of n-ary determiners in general, for arbitrary $n$. This move will allow us to understand better the basic ideas underlying various definitions. Thus most definitions to be given concern type $\left\langle 1^{n}, 1\right\rangle$ quantifiers, that is binary relations whose first argument is an element of an n-ary relation between sets and the second argument is a set.

Most of the definitions we will use have already been suggested (cf. Keenan and Moss 1984, Beghelli 1994, Keenan 2003, Zuber 2005). After the discussion from the previous section we have a relatively clear intuition of how to define conservative quantifiers of higher types. Here are two general definitions: the 
definition of conservative quantifiers and the definition of straightforwardly related generalised cardinals (Zuber 2005):

D10: $D \in C O N S_{\left\langle 1^{n}, 1\right\rangle}$ iff $\forall X_{i}, Y_{1}, Y_{2}, D\left(X_{1}, \ldots, X_{n}\right)\left(Y_{1}\right)=D\left(X_{1}, \ldots, X_{n}\right)\left(Y_{2}\right)$ if $X_{i} \cap Y_{1}=X_{i} \cap Y_{2}$, for every $1 \leq i \leq n$.

D11: $D \in G C A R D_{\left\langle 1^{n}, 1\right\rangle}$ iff $\forall X_{i}, Y_{1}, Y_{2}, D\left(X_{1}, \ldots, X_{n}\right)\left(Y_{1}\right)=D\left(X_{1}, \ldots, X_{n}\right)\left(Y_{2}\right)$ if $\left|X_{i} \cap Y_{1}\right|=\left|X_{i} \cap Y_{2}\right|$, for every $1 \leq i \leq n$.

As in the case of simple quantifiers, conservative type $\left\langle 1^{n}, 1\right\rangle$ quantifiers can be defined in two other ways. This is indicated by the following facts;

Fact 13: $D \in C O N S_{\left\langle 1^{n}, 1\right\rangle}$ iff $D\left(X_{1}, \ldots, X_{2}\right)(Y)=D\left(X_{1}, \ldots, X_{n}\right)\left(Y \cap \bigcup_{n} X_{i}\right)$, for every $1 \leq i \leq n$.

Fact 14: $D \in C O N S_{\left\langle 1^{n}, 1\right\rangle}$ iff $D\left(X_{1}, \ldots, X_{2}\right)(Y)=D\left(X_{1}, \ldots, X_{n}\right)\left(Y \cup \bigcap_{n} X_{i}^{\prime}\right)$, for every $1 \leq i \leq n$.

Notice that conservativity of type $\left\langle 1^{n}, 1\right\rangle$ quantifiers does not mean that we can replace the predicative argument by its meet with subject forming arguments without changing the truth-value. In particular the conservativity of the quantifier denoted by more... than... (thus for $n=2$ ) means that (2a) is equivalent to $(2 \mathrm{~b})$ and not to $(2 \mathrm{c})$ :

(2a) More females than males are students.

(2b) More females than males are among female or male students.

(2c) More females than males are male and female students.

As in the case of simple quantifiers it is not difficult to establish that:

Fact 15: $G C A R D_{\left\langle 1^{n}, 1\right\rangle} \subset C O N S_{\left\langle 1^{n}, 1\right\rangle}$

Both sets, GCARD $D_{\left\langle 1^{n}, 1\right\rangle}$ and $C O N S_{\left\langle 1^{n}, 1\right\rangle}$, form atomic Boolean algebras. More specifically we have (Zuber 2005):

Proposition 16: Let $1 \leq i \leq n, P_{i} \subseteq E$ and $P \subseteq \bigcup_{i} P_{i}$. Then the function $F_{P_{1}, \ldots, P_{n}, P}$ such that $F_{P_{1}, \ldots, P_{n}, P}\left(X_{1}, \ldots, X_{n}\right)(Y)=1$ iff $X_{i}=P_{i}$ and $P=Y \cap \bigcup_{i} X_{i}$ is an atom of $C O N S_{\left\langle 1^{n}, 1\right\rangle}$. All atoms of $C O N S_{\left\langle 1^{n}, 1\right\rangle}$ are of this form.

Comparing the above definitions with the definitions of intersective, cointersective, cardinal and co-cardinal simple quantifiers we see how to define higher type intersective, co-intersecive, cardinal and co-cardinal quantifiers of type $\left\langle 1^{n}, 1\right\rangle$. Thus:

D 12: $D \in I N T_{\left\langle 1^{n}, 1\right\rangle}$ iff $\forall X_{i}, Y_{i}, Z_{1}, Z_{2}$, if $X_{i} \cap Z_{1}=Y_{i} \cap Z_{2}$ then $D\left(X_{1}, \ldots, X_{n}\right)\left(Z_{1}\right)=$ $D\left(Y_{1}, \ldots, Y_{n}\right)\left(Z_{2}\right)$, for every $1 \leq i \leq n$. 
D 13: $D \in C O-I N T_{\left\langle 1^{n}, 1\right\rangle}$ iff $\forall X_{i}, Y_{i}, Z_{1}, Z_{2}$, if $X_{i}-Z_{1}=Y_{i}-Z_{2}$, for every $1 \leq i \leq n$ then $D\left(X_{1}, \ldots, X_{n}\right)\left(Z_{1}\right)=D\left(Y_{1}, \ldots, Y_{n}\right)\left(Z_{2}\right)$

D14: $D \in C A R D_{\left\langle 1^{n}, 1\right\rangle}$ iff $\forall X_{i}, Y_{i}, Z_{1}, Z_{2}$, if $\left|X_{i} \cap Z_{1}\right|=\left|Y_{i} \cap Z_{2}\right|$, for every $1 \leq i \leq n$ then $D\left(X_{1}, \ldots, X_{n}\right)\left(Z_{1}\right)=D\left(Y_{1}, \ldots, Y_{n}\right)\left(Z_{2}\right)$

D15: $D \in C O-C A R D_{\left\langle 1^{n}, 1\right\rangle}$ iff $\forall X_{i}, Y_{i}, Z_{1}, Z_{2}, D\left(X_{1}, \ldots, X_{n}\right)\left(Z_{1}\right)=D\left(Y_{1}, \ldots, Y_{n}\right)\left(Z_{2}\right)$ if $\left|X_{i}-Z_{1}\right|=\left|Y_{i}-Z_{2}\right|$, for every $1 \leq i \leq n$.

Various classes of quantifiers specified in these definitions are related between themselves. We have in particular (Keenan and Moss 1984, Zuber 2005):

Fact 17: $C O N S_{\left\langle 1^{n}, 1\right\rangle}, G C A R D_{\left\langle 1^{n}, 1\right\rangle}, I N T_{\left\langle 1^{n}, 1\right\rangle}, C O-I N T_{\left\langle 1^{n}, 1\right\rangle}, C A R D_{\left\langle 1^{n}, 1\right\rangle}$, $C O-C A R D_{\left\langle 1^{n}, 1\right\rangle}$ form Boolean algebras.

Fact 18: $C A R D_{\left\langle 1^{n}, 1\right\rangle} \subseteq I N T_{\left\langle 1^{n}, 1\right\rangle} \subseteq C O N S_{\left\langle 1^{n}, 1\right\rangle}$

Another relation (holding in finite models) which is easy to establish, and which is analogous to that established in the previous section concerning quantifiers of type $\langle 1,1\rangle$, is indicated in:

Fact 19: $C A R D_{\left\langle 1^{n}, 1\right\rangle} \cup C O-C A R D_{\left\langle 1^{n}, 1\right\rangle} \subseteq G C A R D_{\left\langle 1^{n}, 1\right\rangle} \subseteq C O N S_{\left\langle 1^{n}, 1\right\rangle}$

To be more precise, the above set inclusions can in fact be replaced by statements indicating that included sets are sub-algebras of sets in which they are included.

It is easy to check that the quantifier MORE...THAN... denoted by the binary determiner more ...than... (occurring in NPs on the subject position) is a type $\left\langle 1^{2}, 1\right\rangle$ cardinal quantifier. We will discuss many other examples in the next section.

All the above definitions specify various classes of type $\left\langle 1^{n}, 1\right\rangle$ quantifiers. Natural languages also have clear cases of type $\left\langle 1,1^{n}\right\rangle$ quantifiers or at least of type $\left\langle 1,1^{2}\right\rangle$. An example of such a quantifier is the quantifier MORE...ARE...THAN... (as the denotation of the determiner occurring in More students are Buddhists than shogi players. This means that various classes of type $\left\langle 1,1^{n}\right\rangle$ quantifiers should be distinguished and defined as well. We give here only definitions of conservativity and intersectivity for such quantifiers. These definitions, in conjunction with other definitions given above show how to define other properties of type $\left\langle 1,1^{n}\right\rangle$ quantifiers (cf. Zuber 2005):

D16: $D \in C O N S_{\left\langle 1,1^{n}\right\rangle}$ iff for all $X, Y_{i}, Z_{i}$ if $X \cap Y_{i}=X \cap Z_{i}$, for every $1 \leq i \leq n$ then $D(X)\left(Y_{1}, \ldots, Y_{n}\right)=D(X)\left(Z_{1}, \ldots, Z_{n}\right)$

D17: $D \in I N T_{\left\langle 1,1^{n}\right\rangle}$ iff for all $X_{1}, X_{2}, Y_{i}, Z_{i}, D\left(X_{1}\right)\left(Y_{1}, \ldots, Y_{n}\right)=D\left(X_{2}\right)\left(Z_{1}, \ldots, Z_{n}\right)$, whenever $X_{1} \cap Y_{i}=X_{2} \cap Z_{i}$, for every $1 \leq i \leq n$.

The following fact shows that conservativity for type $\left\langle 1,1^{n}\right\rangle$ quantifiers can be defined in an equivalent way: 
Fact 20: A type $\left\langle 1,1^{n}\right\rangle$ quantifier $D$ is conservative iff for any $X, Y_{i} \subseteq E$ one has $D(X)\left(Y_{1}, \ldots, Y_{n}\right)=D(X)\left(Y_{1} \cap X, \ldots, Y_{n} \cap X\right)$.

When we compare definition D10 of conservativity for type $\left\langle 1^{n}, 1\right\rangle$ quantifiers with the definition D16 of conservativity for type $\left\langle 1,1^{n}\right\rangle$ quantifiers and fact 13 with fact 20 we observe that they do not correspond to the same truth conditions. This means that the cardinality of the set of conservative type $\left\langle 1^{n}, 1\right\rangle$ quantifiers is not the same as the cardinality of the set of conservative type $\left\langle 1,1^{n}\right\rangle$ quantifiers. In particular (cf. Beghelli 1994) in the universe with $n$ individuals there is $2^{7^{n}}$ of type $\left\langle 1^{2}, 1\right\rangle$ conservative quantifiers and $2^{5^{n}}$ of type $\left\langle 1,1^{2}\right\rangle$ conservative quantifiers.

We want now to define symmetry and contraposition for quantifiers of higher types. Obviously, in such definitions we cannot just permute nominal and verbal arguments of the corresponding relation since in this case such a permutation would change the type of quantifier. Consequently we cannot define symmetric higher type quantifiers by comparing relations with permuted arguments as in definition D8 for simple quantifiers. It is possible, however, in this case to use the equivalence indicated in Proposition 11 and 12 and define symmetric and contrapositional quantifiers of higher types in the definitional format mostly used here (Zuber 2007):

D 19: A type $\left\langle 1^{n}, 1\right\rangle$ quantifier $D$ is symmetric iff there exists a binary commutative function $\otimes$ on pairs of sets such that $\forall X_{i}, Y_{i}, Z_{1}, Z_{2}, D\left(X_{1}, \ldots, X_{n}\right)\left(Z_{1}\right)=$ $D\left(Y_{1}, \ldots, Y_{n}\right)\left(Z_{2}\right)$ if $X_{i} \otimes Z_{1}=Y_{i} \otimes Z_{2}$, for every $1 \leq i \leq n$.

D 20: A type $\left\langle 1^{n}, 1\right\rangle$ quantifier $D$ is contrapositional iff there exists a binary commutative function $\otimes$ such that $\forall X_{i}, Y_{i}, Z_{1}, Z_{2}$, if $X_{i} \otimes Z_{1}^{\prime}=Y_{i} \otimes Z_{2}^{\prime}$ then $D\left(X_{1}, \ldots, X_{n}\right)\left(Z_{1}\right)=D\left(Y_{1}, \ldots, Y_{n}\right)\left(Z_{2}\right)$, for every $1 \leq i \leq n$.

Similarly for type $\left\langle 1,1^{n}\right\rangle$ quantifiers:

D 21: A type $\left\langle 1,1^{n}\right\rangle$ quantifier is symmetric iff there exists a binary commutative function $\otimes$ on pairs of sets such that for all $X_{1}, X_{2}, Y_{i}, Z_{i}$, if $X_{1} \otimes Y_{i}=$ $X_{2} \otimes Z_{i}$, then $D\left(X_{1}\right)\left(Y_{1}, \ldots, Y_{n}\right)=D\left(X_{2}\right)\left(Z_{1}, \ldots, Z_{n}\right)$, for every $1 \leq i \leq n$.

D 22: A type $\left\langle 1,1^{n}\right\rangle$ quantifier is contrapositional iff there exists a binary commutative function $\otimes$ on pairs of sets such that for all $X_{1}, X_{2}, Y_{i}, Z_{i}$, if $X_{1} \otimes Y_{i}^{\prime}=$ $X_{2} \cap Z_{i}^{\prime}$, for every $1 \leq i \leq n$ then $D\left(X_{1}\right)\left(Y_{1}, \ldots, Y_{n}\right)=D\left(X_{2}\right)\left(Z_{1}, \ldots, Z_{n}\right)$.

The following propositions partially justify the above definitions of higher type symmetric or contrapositional quantifiers (Zuber 2007):

Proposition 21: Let $F \in P D E T_{\left\langle 1^{n}, 1\right\rangle}$ and $G \in P D E T_{\left\langle 1,1^{n}\right\rangle}$ such that $F\left(X_{1}, \ldots, X_{n}\right)(Y)=$ $G(Y)\left(X_{1}, \ldots, X_{n}\right)$. Then $F$ is symmetric iff $G$ is symmetric.

Thus, roughly, proposition 21 says that if two functions have "symmetric types" and are equal then they are both symmetric. 
From now on, we will talk about higher type quantifiers which are denotations of binary determiners, that is we will suppose that $n=2$. Most of the discussed properties will be explicitly given for type $\left\langle 1^{2}, 1\right\rangle$ quantifiers though it will be obvious that they also hold for the "symmetric" type $\left\langle 1,1^{2}\right\rangle$.

Definitions D19 and D20 are "ineffective" in the sense that they involve quantification over binary commutative functions. They can be simplified due to the following observation (for which I am indebted to Makoto Kanazawa, pc.). Consider binary operation $\ominus$ on ordered pairs of sets defined as follows: $X \ominus Y=\{X, Y\}$. This commutative operation is minimal in the following sense: for any commutative binary operation $\otimes$ the equality $X_{1} \ominus Y_{1}=X_{2} \ominus Y_{2}$ entails the equality $X_{1} \otimes Y_{1}=X_{2} \otimes Y_{2}$. It follows from this that in the definition of symmetry or contraposition we can use just the minimal operation defined above. Consequently we have the following "simplified" but equivalent definition of symmetric type $\left\langle 1^{2}, 1\right\rangle$ quantifiers:

D23: A type $\left\langle 1^{2}, 1\right\rangle$ quantifier $F$ is symmetric iff for all $X_{1}, X_{2}, Y_{1}, Y_{2}, Z_{1}, Z_{2}$, if $\left\{X_{1}, Z_{1}\right\}=\left\{Y_{1}, Z_{2}\right\}$ and $\left\{X_{2}, Z_{1}\right\}=\left\{Y_{2}, Z_{2}\right\}$ then $F\left(X_{1}, X_{2}\right)\left(Z_{1}\right)=F\left(Y_{1}, Y_{2}\right)\left(Z_{2}\right)$

This simplified definition allows us to prove easily various properties of symmetric quantifiers. First, symmetry is preserved under the permutation of nominal arguments. Call $\operatorname{Per}(F)$ a permutation of a type $\left\langle 1^{2}, 1\right\rangle$ quantifier $F$ the quantifier defined as: $\operatorname{Perm}(F)\left(X_{1}, X_{2}\right)(Y)=F\left(X_{2}, X_{1}\right)(Y)$. Then:

Fact 22: A type $\left\langle 1^{2}, 1\right\rangle$ quantifier $F$ is symmetric iff $\operatorname{Perm}(F)$ is symmetric.

We can also now easily prove the following proposition which allows us to understand what symmetry for type $\left\langle 1^{2}, 1\right\rangle$ quantifiers means:

Proposition 23: A type $\left\langle 1^{2}, 1\right\rangle$ quantifier $F$ is symmetric iff for any set $X, Y$ one has $F(X, X)(Y)=F(Y, Y)(X)$.

Proof:

if-part: Suppose $F$ is symmetric. Since for any $X, Y \subseteq E$ one has $\{X, Y\}=$ $\{Y, X\}$, we have $F(X, X)(Y)=F(Y, Y)(X)$.

only-if-part: Suppose $F(X, X)(Y)=F(Y, Y)(X)$. Let (i) hold: (i) $\left\{X_{1}, Z_{1}\right\}=$ $\left\{Y_{1}, Z_{2}\right\}$ and $\left\{X_{2}, Z_{1}\right\}=\left\{Y_{2}, Z_{2}\right\}$. We consider four cases in which equalities in (i) hold:

(1) $X_{1}=Y_{1}, Z_{1}=Z_{2}$, and $X_{2}=Y_{2}$. In this case trivially $F\left(X_{1}, X_{2}\right)\left(Z_{1}\right)=$ $F\left(Y_{1}, Y_{2}\right)\left(Z_{2}\right)$.

(2) $X_{1}=Y_{1}, Z_{1}=Z_{2}, X_{2}=Z_{2}$ and $Z_{1}=Y_{2}$. Thus $X_{2}=Y_{2}=Z_{1}=Z_{2}$ and $X_{1}=Y_{1}$ and consequently $F\left(X_{1}, X_{2}\right)\left(Z_{1}\right)=F\left(Y_{1}, Y_{2}\right)\left(Z_{2}\right)$.

(3) $X_{1}=Z_{2}, Z_{1}=Y_{1}, X_{2}=Y_{2}$ and $Z_{1}=Z_{2}$. Thus $X_{1}=Y_{1}=Z_{1}=Z_{2}$ and $X_{2}=Y_{2}$ and consequently $F\left(X_{1}, X_{2}\right)\left(Z_{1}\right)=F\left(Y_{1}, Y_{2}\right)\left(Z_{2}\right)$.

(4) $X_{1}=Z_{2}, Z_{1}=Y_{1}, X_{2}=Z_{2}$ and $Z_{1}=Y_{2}$. Thus $X_{1}=X_{2}=Z_{2}$ and $Y_{1}=Y_{2}=Z_{1}$. From this and from the supposition it follows that $F\left(X_{1}, X_{2}\right)\left(Z_{1}\right)=F\left(Y_{1}, Y_{2}\right)\left(Z_{2}\right)$. 
Using Proposition 23 one shows that symmetric quantifiers form a Boolean algebra with Boolean operations defined pointwise.

The property indicated in Proposition 24 concerns symmetric quantifiers in general, not necessarily conservative ones. Since the operation of the meet on sets is a commutative we have obviously the following fact:

Fact 24: If $F \in I N T_{\left\langle 1^{2}, 1\right\rangle}$ or $F \in I N T_{\left\langle 1,1^{2}\right\rangle}$ then $F$ is symmetric.

Moreover for conservative symmetric quantifiers the following is true:

Proposition 25: If a type $\left\langle 1^{2}, 1\right\rangle$ quantifier $F$ is conservative then $F$ is symmetric iff $F(X, X)(Y)=F(X \cap Y, X \cap Y)(X \cap Y)$

Notice that in the above proposition symmetry cannot be replaced by intersectivity which means that under conservativity symmetry and intersectivity do not coincide, as is the case for simple quantifiers. Thus there are conservative quantifiers which are symmetric but not intersective. Here are two examples of such quantifiers.

First consider the quantifier $F$ defined as follows: $F\left(X_{1}, X_{2}\right)(Y)=1$ iff $X_{1} \cap X_{2} \neq \emptyset$ and $\left|X_{1} \cap Y\right|=\left|X_{2} \cap Y\right| \neq 0$. One can check that this quantifier is conservative and symmetric but not intersective.

As a second example consider atoms of conservative type $\left\langle 1^{2}, 1\right\rangle$ quantifiers. Recall that according to proposition 16 atoms of conservative type $\left\langle 1^{2}, 1\right\rangle$ quantifiers are functions $F_{A_{1}, A_{2}, B}$ such that $B \subseteq A_{1} \cup A_{2}$ and $F_{A_{1}, A_{2}, B}\left(X_{1}, X_{2}\right)(Y)=$ 1 iff $A_{i}=X_{i}$ and $B=Y \cap\left(A_{1} \cup A_{2}\right)$. One can show now, using the simplified definition of symmetry, that such atomic functions are symmetric if $A_{1} \cup A_{2}=E$ and $A_{1} \neq A_{2}$ and among them the intersective ones are only those for which $B=E$.

In fact the quantifiers indicated in the second example above constitute atoms of the algebra of conservative symmetric quantifiers. More specifically:

Proposition 26: Conservative symmetric type $\left\langle 1^{2}, 1\right\rangle$ quantifiers form an atomic Boolean algebra. For any set $A_{1}, A_{2}, B$ such that $A_{1} \cup A_{2}=E$ functions $F_{A_{1}, A_{2}, B}$ such that $F_{A_{1}, A_{2}, B}\left(X_{1}, X_{2}\right)(Y)=1$ iff (if $A_{1} \neq A_{2}$ then $A_{i}=X_{i}$ and $Y=B$ ) and (if $A_{1}=A_{2}$ then $A_{i}=X_{i}$ and $Y=E$ ) are atoms of this algebra.

Proposition 26 allows us to calculate the number of symmetric type $\left\langle 1^{2}, 1\right\rangle$ quantifiers. One calculates that in the universe with $n$ individuals there are $6^{n}-2^{n}+1$ atomic symmetric and conservative type $\left\langle 1^{2}, 1\right\rangle$ quantifiers. So in particular in the universe with 2 elements we have $2^{33}$ symmetric type $\left\langle 1^{2}, 1\right\rangle$ quantifiers among which $2^{16}$ are intersective. The number of all type $\left\langle 1^{2}, 1\right\rangle$ conservative quantifiers equals in this case $2^{49}$ and the number of all "unrestricted" type $\left\langle 1^{2}, 1\right\rangle$ quantifiers equals $2^{64}$.

In the next section we will see some binary determiners denoting conserva- 
tive symmetric but not intersective quantifiers.

There remain some important issues to be mentioned concerning contrapositional quantifiers and their relationship to symmetric ones. Recall that in the case of simple conservative quantifiers we have different classes of contrapositional and symmetric quantifiers. They are respectively intesective and co-intersective and only constant simple conservative quantifiers are at the same time intersective and co-intersective. The situation is quite different in the case of higher type quantifiers since it appears that in this case the distinction between symmetric and contrapositional quantifiers is not very interesting. Let us see this in the case of type $\left\langle 1^{2}, 1\right\rangle$ quantifiers.

Observe first that, given that the equality $\{X, Y\}=\left\{X, Y^{\prime}\right\}$ never holds, we have the following property:

Proposition 27: If $F$ is symmetric or contrapositional type $\left\langle 1^{2}, 1\right\rangle$ quantifier then for any $X, Y \in E$ the following holds: $F(X, X)(Y)=F(X, X)\left(Y^{\prime}\right)$

Proposition 27 allows us to prove the following important fact:

Proposition 28: $F$ is symmetric type $\left\langle 1^{2}, 1\right\rangle$ quantifier iff $F$ is contrapositional.

Thus the distinction between symmetric and contrapositional quantifiers has no theoretic basis in the case of higher type quantifiers.

The last class of type $\left\langle 1^{2}, 1\right\rangle$ quantifiers that we will mention is the class of proportional quantifiers. They are defined as follows (Zuber 2005):

D24: $D \in P R O P O R T_{\left\langle 1^{2}, 1\right\rangle}$ iff for all $X_{1}, X_{2}, Y_{1}, Y_{2}, Z_{1}, Z_{2}, D\left(X_{1}, X_{2}\right)\left(Z_{1}\right)=$ $D\left(Y_{1}, Y_{2}\right)\left(Z_{2}\right)$ whenever $\left|Y_{1}\right| \times\left|Y_{2}\right| \times\left|X_{1} \cap Z_{1}\right|=\left|X_{1}\right| \times\left|X_{2}\right| \times\left|Y_{1} \cap Z_{2}\right|$ and $\left|Y_{1}\right| \times\left|Y_{2}\right| \times\left|X_{2} \cap Z_{1}\right|=\left|X_{1}\right| \times\left|X_{2}\right| \times\left|Y_{2} \cap Z_{2}\right|$.

One checks by calculation that according to D 24 determiners like proportionally as many... as... denote proportional type $\left\langle 1^{2}, 1\right\rangle$ quantifiers.

For proportional quantifiers the following is true (Zuber 2005):

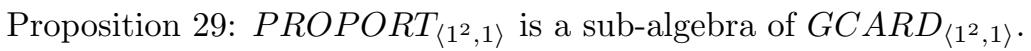

To conclude this section let me mention some differences between simple and higher type quantifiers. One important difference concerns simple and binary proportional quantifiers; only the former are closed with respect to post-complement. Other differences, more important for our analysis, concern intersective and symmetric quantifiers. We have seen that under conservativity these two notions are co-extensive. Furthermore, we have also seen that the intersectivity of simple quantifiers can be defined in four equivalent ways (cf. Fact 4$)$. This is not the case for intersectivity of type $\left\langle 1^{2}, 1\right\rangle$ or type $\left\langle 1,1^{2}\right\rangle$ quantifiers. In particular there are symmetric conservative type $\left\langle 1^{2}, 1\right\rangle$ quantifieres which are not intersective. Finally, we observe that in the case of higher 
type quantifiers the class of symmetric quantifiers coincides with the class of contrapositional quantifiers.

\section{Denotations of binary determiners}

As already indicated in the introduction, binary determiners are discontinuous functional expressions which take two arguments. Syntactically, they are not necessarily "nominal" because their arguments can be of two categories. First, they can take two common nouns and form a noun phrase. Though such NPs can occur on various positions we will consider only the case when they occur in subject position. Thus determiners of the first category form a sentence with two common nouns and a verb phrase. Consequently, semantically, they denote type $\left\langle 1^{2}, 1\right\rangle$ quantifiers. Second, binary determiners can take two verb phrases and form with one common noun a sentence. In this case they denote $\left\langle 1,1^{2}\right\rangle$ quantifiers. Our proposal here concerns basically binary determiners of the first category.

From the formal point of view it is useful to distinguish two types of quantifiers denoted by binary (or n-ary) determiners (cf. Keenan and Moss 1985): Booleanly reducible quantifiers and irreducible quantifiers. Reducible quantifiers are defined as follows (Beghelli 1994):

D25: A type $\left\langle 1^{2}, 1\right\rangle$ quantifier $F$ is (Booleanly) reducible iff there exist simple (type $\langle 1,1\rangle)$ quantifiers $Q_{1}$ and $Q_{2}$ and a binary Boolean function $h$ such that $F\left(X_{1}, X_{2}\right)(Y)=h\left(Q_{1}\left(X_{1}\right)(Y), Q_{2}\left(X_{2}\right)(Y)\right)$

Determiners which denote reducible (irreducible) quantifiers will also be called reducible (irreducible).

We will say that $Q_{1}$ and $Q_{2}$ are Boolean components of the reducible quantifier $F$. In the simplest case $Q_{1}=Q_{2}$ and in practice $F$ has often the same lexical form as its component $Q$ (but not the same category). For instance numerals, which basically denote simple quantifiers, can also be considered as denoting reducible type $\left\langle 1^{2}, 1\right\rangle$ quantifiers when their first argument is a conjunction or a disjunction of two common nouns. For instance since (3) has a natural reading corresponding to the conjunction in (4), the numeral five can be considered as a binary determiner denoting a reducible quantifier:

(3) Five students and teachers are dancing.

(4) Five students and five teachers are dancing.

Various examples of reducible quantifiers are studied in Keenan and Moss 1985.

It is clear that many properties of reducible quantifiers are induced by their components. In particular we have the following:

Proposition 30: A reducible type $\left\langle 1^{2}, 1\right\rangle$ quantifier $F$ is intersective (in its type) iff its components $Q_{1}$ and $Q_{2}$ are both intersective. 
Given that simple conservative quantifiers are symmetric iff they are intersective we obtain the following important consequence from proposition 30:

Fact 31: Any symmetric reducible type $\left\langle 1^{2}, 1\right\rangle$ quantifier is intersective.

Thus if a type $\left\langle 1^{2}, 1\right\rangle$ quantifier is symmetric but not intersective then it is not reducible. Furthermore, if a reducible quantifier has non intersective components then it can be non symmetric. So we will consider basically non-reducible quantifiers.

Often discussed in the literature "natural" higher type quantifiers are socalled comparative binary quantifiers. These are quantifiers denoted in the simplest case by discontinuous (binary) determiners like more... than or as many... as. As we have already seen, when these determiners form subject NPs (in sentences with "simple" VPs) then they denote quantifiers of type $\left\langle 1^{2}, 1\right\rangle$. These quantifiers can be said to be genuine higher type since they cannot be reduced (in the sense of definition D25) to a Boolean combination of simple quantifiers (Keenan and Moss 1985, Beghelli 1994). Furthermore, they are "natural" in the sense that the determiners by which they are denoted have a categorial status of syntactically justified binary determiners (Keenan 1989).

Beghelli (1994) distinguishes various sub-groups of comparative determiners. Usually they exhibit a complex syntax which can be ignored for our purposes. The simplest and in some sense basic group of determiners may be called simple comparatives. They include determiners like more...than..., exactly as many... as..., the same number of..as.., , etc. It is easy to see that these determiners denote cardinal quantifiers and thus, given fact 18 and fact 24 , they are symmetric and, at the same time, generalised cardinals. Let us show this for illustration on one example. Consider for instance the quantifier $F E W E R \ldots T H A N \ldots$ denoted by the determiner occurring in the noun phrase fewer students than teachers. Its semantics is given in (5):

(5) $F E W E R\left(X_{1}\right) T H A N\left(X_{2}\right)(Y)=1$ iff $\left|X_{1} \cap Y\right|<\left|X_{2} \cap Y\right|$

To show that it is cardinal, suppose that (6) and (7) hold. We have to show that (8) holds as well:

(6) $\left|X_{1} \cap Z_{1}\right|=\left|Y_{1} \cap Z_{2}\right|$ and $\left|X_{2} \cap Z_{1}\right|=\left|X_{2} \cap Z_{2}\right|$

(7) $F E W E R\left(X_{1}\right) T H A N\left(X_{2}\right)\left(Z_{1}\right)=1$

(8) $\operatorname{FEWER}\left(Y_{1}\right) \operatorname{THAN}\left(Y_{2}\right)\left(Z_{2}\right)=1$

The result is obvious given the semantics in (5): the equalities in (6) allow us to make replacements making (8) true.

Since cardinal quantifiers (of any type) form a Boolean algebra, they are closed with respect to Boolean operations. This means for instance that $A T$ $L E A S T-A S-M A N Y \ldots A S \ldots$ is also cardinal because it is the complement of 
FEWER...THAN.... Similarly the complex determiner at least 10 more but not more than 20 more... than ... (as in at least ten more but not more then 20 students more then teachers) denotes the meet of two quantifiers denoted respectively by at least 10 more... than... and not more than 20 ...than.... Since both these quantifiers are cardinal the whole quantifier is also a cardinal and consequently symmetric and generalised cardinal. The symmetry of many other quantifiers can be established in the same way (see Beghelli 1994).

One observes in addition that for many simple comparative determiners mentioned above there exist logically equivalent syntactically more complex ones. For instance a lesser number of... than... is semantically equivalent to fewer...than... and exactly as many... as... is equivalent to exactly the same number of... as.... Their semantic status is however the same.

There is a class of binary determiners which denote intersective but not cardinal quantifiers. These are determiners which may be called modified comparatives. They can be modified by adjectives (as in more male... than female... or by possessives (as in more Leo's... than Bill's...). What is interesting is the fact that though such modified comparatives are in some sense derived from cardinal ones they are not cardinal. In other words the modification does not preserve the property of being cardinal. However, modification preserves intersectivity. Let us see this in more details.

Observe first that the modifiers we are talking about denote absolute functions (absolute modifiers). A function $M$ from sets to sets is absolute (Keenan and Faltz 1985) iff for any set $X, M(X)=X \cap M(E)$. Absolute adjectives (male, female) and (some) possessives denote absolute modifiers. Thus, roughly female students are students and female objects and Bill's bicycles are bicycles and Bill's objects.

Let us define now an intersective type $\left\langle 1^{2}, 1\right\rangle$ quantifier restricted (modified) by two sets:

D26: Let $A$ and $B$ be sets and $D$ a type $\left\langle 1^{2}, 1\right\rangle$ quantifier. Then $D_{A, B}$ is a type $\left\langle 1^{2}, 1\right\rangle$ quantifier defined as follows: $D_{A, B}\left(X_{1}, X_{2}\right)(Y)=D\left(A \cap X_{1}, B \cap X_{2}\right)(Y)$

For such modified quantifiers it is easy to establish the following fact:

Fact 32: If $D \in I N T_{\left\langle 1^{2}, 1\right\rangle}$ then $D_{A, B} \in I N T_{\left\langle 1^{2}, 1\right\rangle}$, for any set $A, B$.

It follows from fact 32 that modified comparative binary determiners denote intersective quantifiers (because they are obtained by the modification of cardinal, and thus of intersective quantifiers). Consequently modified comparative binary determiners also denote symmetric (but not generalised cardinal) quantifiers.

Beghelli (1994) also mentions existence of the class of binary determiners he calls identity comparative. These determiners do not involve comparison of cardinalities or quantities but rather a comparison of identities of individuals. Syntactically, they combine one common noun with two VPs to form a sen- 
tence and thus they denote type $\left\langle 1,1^{2}\right\rangle$ quantifiers. Here are some (Beghelli's) examples of sentences with such determiners:

(9) The same students came early as left late.

(10) Whatever students came early left late.

(11) The same five students came early as left late.

The determiners in the above sentences denote the following quantifiers respectively:

(12) THE-SAME $(X)\left(Y_{1}, Y_{2}\right)=1$ iff $X \cap Y_{1}=X \cap Y_{2}$

(13) WHATEVER(X)(Y $\left.Y_{1}, Y_{2}\right)=1$ iff $X \cap Y_{1} \subseteq X \cap Y_{2}$

(14) THE-SAME-5(X)(Y, $\left.Y_{2}\right)=1$ iff $X \cap Y_{1}=X \cap Y_{2} \wedge\left|X \cap Y_{1}\right|=5$

Thus sentence (10) is true iff the set of students who came early is included in the set of students who left late. It is easy to show that quantifiers in (12), (13) and (14) are all intersective and thus symmetric.

Recall that type $\left\langle 1,1^{2}\right\rangle$ quantifiers can also be denoted by "ordinary" comparative determiners as in the following examples:

(15) More vegetarians are students than teachers.

(16) More students came early than left early.

The binary determiners in these examples denote intersective, and thus symmetric quantifiers.

There remains a last type of determiners we need to examine. It is represented by the proportional binary determiners as the one found in (17). It denotes a type $\left\langle 1^{2}, 1\right\rangle$ quantifier which has the semantics given in (18):

(17) Proportionally as many students as teachers danced.

(18) PROP-AS-MANY $\left(X_{1}, X_{2}\right)(Y)=1$ iff $\left|X_{1} \cap Y\right| /\left|X_{1}\right|=\left|X_{2} \cap Y\right| /\left|X_{2}\right|$

The quantifier in (18) is proportional in the sense of D24. It is not intersective. We show, not quite explicitly, that it is symmetric. According to D19 we have to show that there exists a binary operation on sets $\otimes$ which is commutative and such that if (19) holds then (20) holds:

(19) $X_{1} \otimes Z_{1}=Y_{1} \otimes Z_{2}$ and $X_{2} \otimes Z_{1}=Y_{2} \otimes Z_{2}$

(20) PROP-AS-MANY $\left(X_{1}, X_{2}\right)\left(Z_{1}\right)=P R O P-A S-M A N Y\left(Y_{1}, Y_{2}\right)\left(Z_{2}\right)$

Chose $\otimes$ as: $X \otimes Y=|X \cap Y| /(|X| \times|Y|)$. This operation is obviously commutative. A somewhat tedious simple arithmetic operation on fractions in conjunction with necessary substitutions of equals by equals leads to the required equivalence in (20). 
One shows in the same way that determiners like proportionally more...than... denote symmetric quantifiers.

In addition, the symmetry of some quantifiers can be established using fact 22 , that is the fact that the permutation of nominal arguments preserves symmetry. For instance since the quantifier denoted by the determiner proportionally more...than... is related by the relation of permutation to the quantifier denoted by proportionally less...than..., the symmetry of one follows from the symmetry of the other. Thus proportional comparative determiners also denote (conservative) symmetric quantifiers.

\section{Conclusion}

In this paper I have been concerned with the constraint of symmetry on quantifiers which are denotations of binary determiners. Symmetric type $\left\langle 1^{2}, 1\right\rangle$ quantifiers $F$ are those and only those quantifiers which have the property indicated in (21a); they entail the property indicated in (21b):

(21a) $F(X, X)(Y)=F(Y, Y)(X)$

(21b) $F(X, X)(Y)=F(X, X)\left(Y^{\prime}\right)$

So the claim defended here entails that all irreducible binary determiners denote quantifiers which satisfy (21a) and (21b). And this indeed seems to be the case.

Obviously not all type $\left\langle 1^{2}, 1\right\rangle$ quantifiers have such properties and the constraint of symmetry severely constrains the number of possible denotations for binary determiners. This constraint makes unary determiners different from binary ones. More specifically in this paper, using the generalised notion of symmetry, I insisted on the following difference: under conservativity not all symmetric type $\left\langle 1^{2}, 1\right\rangle$ quantifiers are intersective. It was shown in this context that all proper (that is Booleanly irreducible) binary determiners discussed in literature denote conservative symmetric quantifiers. Roughly speaking binary comparative determiners denote cardinal quantifiers. They are symmetric precisely because being cardinal they are intersective. Some comparative binary determiners, those where their arguments are modified by absolute adjectives, are not cardinal but they are still intersective (cf. fact 32). Finally proportional comparative determiners denote symmetric (and conservative) type $\left\langle 1^{2}, 1\right\rangle$ quantifiers which are not intersective.

We also considered from the point of view of symmetry, so-called identity comparative determiners. They are syntactically different from other comparative determiners since they form a sentence with one common noun and two verb phrases. They denote intersective type $\left\langle 1,1^{2}\right\rangle$ quantifiers and thus are also symmetric. We also indicated that some "ordinary" comparative binary determiners can denote irreducible type $\left\langle 1,1^{2}\right\rangle$ quantifiers which are symmetric.

It is important to keep in mind that the claim defended here concerns binary determiners denoting irreducible quantifiers. One observes for instance that the determiner most when applied to a conjunction of two common nouns 
can have a reading under which it denotes a type $\left\langle 1^{2}, 1\right\rangle$ quantifier. Thus $(22 \mathrm{a})$ has a natural reading under which it is equivalent to $(22 \mathrm{~b})$ :

(22a) Most students and teachers danced.

(22b) Most students danced and most teachers danced.

It is easy to show that under this reading most denotes a conservative type $\left\langle 1^{2}, 1\right\rangle$ quantifier which is not symmetric. But this quantifier is Booleanly reducible and thus is not a counter-example to the claim.

Notice in this context that the claim defended here is obviously meant to be language independent. We know that there are cross-linguistic variations concerning the possibility for unary determiners to have their type shifted to the type of binary determiners in some conjunctive expressions. So the restriction of the claim to irreducible binary determiners, which seem less language dependent, should not be surprising.

When speaking about counter-examples let me mention another claim which could be made about the semantic constraint on the denotations of binary determiners. Given the various properties discussed above, one might be tempted to claim that binary determiners always denote generalised cardinals. Indeed cardinal quantifiers denoted by comparative determiners are generalised cardinals. Similarly with proportional comparative determiners: they also denote generalised cardinals. There are, however, two classes of counter-examples. First, identity comparatives denote intersective quantifiers and intersective quantifiers are not generalised cardinals. Maybe the fact that such determiners are syntactically different from others might be used to save the clam by reducing its domain to binary determiners denoting only type type $\left\langle 1^{2}, 1\right\rangle$ quantifiers. There is, however another class of counter-examples: these are modified comparative determiners like more male... than female... They denote intersective type $\left\langle 1^{2}, 1\right\rangle$ quantifiers which are not generalised cardinals.

Let me conclude with the following negatively oriented remark: what I have done up to now can be considered as just an empirical generalisation based on various examples of determiners, most of which are known from the relevant literature. Two important things remain to be done. First, the claim has to be explained and some reasons why binary determiners denote symmetric quantifiers given. Second, how the claim is related to various aspects of expressibility in natural languages. Any attempt to answer such questions necessitates additional technical notions such as universe independence, relativization of type $\langle 1\rangle$ quantifiers, etc. Peters and Westerståhl 2006 discuss symmetry and related notions of simple quantifiers in the context of these additional notions. Their extensions and various generalisations are needed to get some answers to such general questions.

\section{References}

[1] Beghelli, F. (1992) Comparative Quantifiers, in Dekker, P. and Stokhof, 
M. (eds.) Proc. of the VIII Amsterdam Colloquium

[2] Beghelli, F. (1994) Structured Quantifiers, in Kanazawa, M. and Piñon, Ch. (eds.) Dynamics, Polarity, and Quantification, CSLI Publications, $119-145$

[3] Keenan, E. L. (1987) Multiply-headed NPs, Linguistic Inquiry 18, pp. 481-491

[4] Keenan, E. L. (1993) Natural Language, Sortal Reducibility and Generalised Quantifiers, Journal of Symbolic Logic 58:1, pp. 314-325.

[5] Keenan, E.L. (2002) Some Properties of Natural Language Quantifiers: Generalized Quantifier Theory, Linguistics and Philosophy 25:5-6, pp.627654

[6] Keenan, E. L. and Faltz, L. M. (1985) Boolean Semantics for Natural Language, D. Reidel Publishing Company, Dordrecht.

[7] Keenan, E. L. and Moss, L. (1985) Generalized quantifiers and the expressive power of natural language, in J. van Benthem and A. ter Meulen (eds.) Generalized Quantifiers, Foris, Dordrecht, pp.73-124

[8] Keenan, E. L. and Stavi, J. (1986) A semantic characterisation of natural language determiners, Linguistics and Philosophy 9, pp.253-326

[9] Keenan, E. L. and Westerståhl, D. (1997) Generalized Quantifiers in Linguistics and Logic, in van Benthem, J. and ter Meulen, A. (eds.) Handbook of Logic and Language, Elsevier, pp. 837-893.

[10] Peters, S. and Westerståhl, D. (2006) Quantifiers in Language and Logic, Clarendon Press, Oxford

[11] Zuber, R. (1998) On the Semantics of Exclusion and Inclusion Phrases, in Lawson, A. (ed.) SALT8, Cornell University Press, pp. 267-283

[12] Zuber, R. (2004a) A class of non-conservative determiners in Polish, Linguisticae Investigationes, XXVII : 1, pp. 147-165

[13] Zuber, R. (2004b) Some remarks on syncategorematicity, in L. Hunyadi et al.: The Eighth Symposium on Logic and Language: Preliminary Papers, Debrecen 2004, pp. 165-174

[14] Zuber, R. (2005) More Algebras for Determiners, in P. Blache and E. Stabler (eds.) Logical Aspects of Computational Linguistics 5, LNAI, vol. 3492, Springer-Verlag, pp. 363-378

[15] Zuber, R. (2007) Symmetric and Contrapositional Quantifiers, Journal of Logic, Language and Information 16:1, pp. 1-13 